\title{
Balkanologie
}

Balkanologie Revue d'études pluridisciplinaires

Vol. VII, $n^{\circ} 1$ | 2003

Volume VII Numéro 1

\section{Les technologies d'information et de communication : support de l'émergence d'une diaspora roumaine?}

Mihaela Nedelcu

\section{OpenEdition}

Journals

Édition électronique

URL : http://journals.openedition.org/balkanologie/468

DOI : 10.4000/balkanologie.468

ISSN : 1965-0582

Éditeur

Association française d'études sur les Balkans (Afebalk)

Édition imprimée

Date de publication : 1 juin 2003

Pagination : 43-63

ISSN : $1279-7952$

Référence électronique

Mihaela Nedelcu, «Les technologies d'information et de communication : support de l'émergence d'une diaspora roumaine? », Balkanologie [En ligne], Vol. VII, nº 1 | 2003, mis en ligne le 18 février 2009, consulté le 17 décembre 2020. URL : http://journals.openedition.org/balkanologie/468 ; DOI : https:// doi.org/10.4000/balkanologie.468 


\title{
LES TECHNOLOGIES D'INFORMATION ET DE COMMUNICATION : SUPPORT DE L'ÉMERGENCE D'UNE DIASPORA ROUMAINE ?
}

\author{
Mihaela Nedelcu*
}

Le tournant du XXème siècle, profondément marqué par les effets de la révolution digitale, correspond à une forte re-modélisation des processus migratoires dans leurs structures et leurs dynamiques. Les technologies d'information et de communication (TIC) engendrent des circulations accrues de professionnels à l'intérieur des marchés globaux (les flux migratoires de spécialistes en technologie de l'information étant centraux aux mobilités des professionnels hautement qualifiés dans le monde ${ }^{1}$ ) et fournissent des outils créatifs de communication, d'organisation et de coopération à distance. La mobilité - géographique et sociale - semble être entrée dans une ère nouvelle car les TIC décentralisent les circulations tout en démocratisant les participations.

Internet, en tant qu'outil de communication et nouveau média, façonne différemment les notions de frontière, d'espace, de temps et de mobilité... mais en quoi concerne-t-il les diasporas ? Dans le contexte des migrations internationales l'espace virtuel remplit des fonctions multiples. Instrument de circulation de l'information, "agora" des minorités, instrument de lutte et d'expression idéologique ${ }^{2}$ mais aussi espace de (pré)socialisation des migrants à caractère performatif 3 , il serait "particulièrement pertinent au sein des diasporas, car décentralisé, interactif et transnational par essence"4.

\footnotetext{
- Sociologue, chercheuse et doctorante en sociologie, Institut de Sociologie de l'Université de Neuchâtel, et Ecole Doctorale en Sciences Sociales d'Europe Centrale. mihaela.nedelcu@unine.ch

1 Selon le rapport International Mobility of the Highly Skilled, OCDE, 2002.

2 Diminescu (Dana), “Les migrations à l'ère des nouvelles technologies”, Hommes \& Migrations, (1240), 2002.

3 Nedelcu (Mihaela), "E-stratégies migratoires et communautaires : le cas des Roumains à Toronto", Hommes \& Migrations, (1240), 2002.
}

4 Georgiou (Myria), "Les diasporas en ligne, une expérience concrète de transnationalisme”, Hommes \& Migrations, (1240), 2002, p. 10. 
Cet article est un questionnement sur la pertinence d'un concept qui a fait ses preuves dans des contextes géopolitiques spécifiques pour décrire des phénomènes migratoires essentiellement nouveaux. À une époque où les flux migratoires connaissent des modélisations et des dynamiques complètement nouvelles, les diasporas, en tant qu'entités socialement construites, se transforment-elles aussi dans leur sens originel ? Les TIC ont-elles un impact réel sur les pratiques diasporiques ? Internet n'est-il qu'accélérateur des processus déjà inscrits dans une longue durée, ou contribue-t-il à l'émergence d'un corps social en diaspora nouveau?

Ces interrogations dans notre contexte de recherche renvoient à une double problématique : nous nous intéressons aux processus de cristallisation diasporique d'une immigration jeune - la migration roumaine postcommuniste au Canada - et, de plus, nous postulons que les pratiques médiatisées par les TIC sont les propulseurs d'une dynamique de type diaspora.

\section{LES DIASPORAS MODERNES OU LA RÉ-OPÉRATIONALISATION D'UN CONCEPT}

Le concept de diaspora a connu depuis une vingtaine d'années une inflation d'usages pour désigner aujourd'hui des processus migratoires très diversifiés. Entrée dans le sens commun, cette notion est employée de manière fortement contrastée par des sociologues, politologues ou géographes. Le paradigme de la diaspora juive et grecque, dans son sens originel, comportait quelques traits incontournables : l'exil sous la contrainte ou la dispersion forcée, une conscience identitaire très forte en lien avec la loyauté non dissimulée aux origines, l'aspiration à un retour réel ou symbolique, une présence dominée et non dominante dans la société d'accueil, le polycentrisme des flux et des noyaux diasporiques 5 . La conscience d'exister en diaspora trouve ses raisons dans la fabrication d'une mémoire et d'une histoire propre, fondatrices de la diaspora. Emmanuel Ma Mung met l'accent sur la spatialité et la temporalité de ce processus :

l'identité sociale, en ce qu'elle s'édifie sur une supposée origine commune, est orientée vers le passé et vers la construction d'une mémoire collective (...). Le temps ainsi maitrisé permet de saisir l'espace, la dispersion : la diaspora. (...) Il

5 Bruneau (Michel), "Espaces et territoires de diasporas", in Bruneau (Michel), éd., Diasporas, Montpellier : Reclus, 1995 ; Cohen (Robin), Global Diasporas. An Introduction, University of Washington Press, 1997. 
donne à penser la diaspora comme une unité spatiale et temporelle, comme un être-ensemble unitaire alors qu'elle est, aussi, dispersion géographique des corps et des mémoires individuelles 6 .

Plusieurs auteurs proposent des typologies qui permettent d'élargir la sphère d'opérationnalisation de ce concept : diasporas sans État (d'origine) ou diasporas basées sur un État7 ; diasporas "victimes", "impériales", "culturelles" ou "économiques" ; diasporas "classiques", "vétéranes", "naissantes" ou "dormantes" 9 . Ces distinctions font preuve de l'usage exhaustif de ce concept pour décrire des processus diasporiques modernes, modelés par des contextes géopolitiques complexes et changeants.

$\mathrm{Au} \mathrm{XXème} \mathrm{siècle,} \mathrm{le} \mathrm{phénomène} \mathrm{de} \mathrm{diaspora} \mathrm{s'est} \mathrm{généralisé} \mathrm{davantage} \mathrm{par}$ la facilité accrue des transports et des communications ${ }^{10}$. Dans une approche plutôt maximaliste, "les diasporas modernes sont des minorités ethniques de migrants vivant dans le pays d'accueil mais conservant des liens affectifs et matériels forts avec leur pays d'origine"11. Il parait, selon cette définition permissive, qu'il suffit de garder des liens multiples avec la matrice d'origine pour former une diaspora. Pourtant, étant donné l'avancée technologique et communicationnelle de nos sociétés, ces liens sont propres à presque toute migration, qu'elle soit de courte ou de longue durée. Que fait alors la spécificité actuelle d'une diaspora? Michel Bruneau insiste sur au moins trois éléments : "la conscience et le fait de revendiquer une identité ethnique ou nationale ; l'existence d'une organisation politique, religieuse ou culturelle du groupe dispersé (richesse de la vie associative) ; l'existence des contacts sous diverses formes, réelles ou imaginaires, avec le territoire ou pays d'origine"12 - soit le communautaire (pays d'accueil), la mémoire des origines (pays d'origine) et le système de relations entre les différents pôles de la migration. Ce dernier se traduit par l'aptitude des diasporas à créer et à maintenir des réseaux complexes et polycentriques, essentiels pour la préservation identitaire et des traditions culturelles ${ }^{13}$.

6 Ma Mung (Emmanuel), "La dispersion comme ressource", Cultures \& Conflits, (33-34), 1999.

7 Sheffer (Gabriel), "Whither the study of ethnic diasporas ? Some theoretical, definitional, analytical and comparative considerations", in Prévélakis (Georges), éd., Les réseaux des diasporas, Paris : 1'Harmattan / KIKEM, 1996.

8 Cf Cohen (Robin), op.cit.

9 Cf. Sheffer (Gabriel), art.cit.

10 Gottmann (Jean), “La généralisation des diasporas et ses conséquences”, in Prévélakis (Georges), éd. op.cit.

11 Sheffer (Gabriel), Modern Diasporas in International Politics, New York : Saint Martin's Press, 1986.

12 Bruneau (Michel), art.cit.

13 Prévélakis (Georges), "Introduction” in Prévélakis (Georges), éd., op.cit. 
L'espace d'une diaspora est donc par excellence un espace transnational discontinu et réticulé, structuré "par une pluralité de réseaux dans lesquels circulent idées, hommes, capitaux, marchandises diverses, les liens familiaux et communautaires étant le support de ces échanges" ${ }^{14}$. Mais tout réseau transnational s'inscrit-il dans une dynamique de type diaspora ? Certains auteurs soulèvent la tendance à positionner épistémologiquement les diasporas et les processus réticulaires transnationaux contemporains dans un dualisme syncrétique $^{15}$. Entités sociales et politiques trans-étatiques, les diasporas s'expriment par deux formes complémentaires de solidarité, le "communalisme" et le "corporatisme" 16 , qui ne sont que le reflet des modèles d'autorité complexe, divisée et duale. Elles répondent "au besoin d'appartenance à une communauté plus large que celle de la localité ou de la région"17. On pourrait se demander si l'État-Nation - utilisé de manière instrumentale par les diasporés qui s'adaptent aux systèmes de mobilités propres à un monde globale - n'est pas mis en question de ce fait ${ }^{18}$. La question n'est pas tranchée, plusieurs facteurs contribuant de nos jours à l'accélération et à la complexité des dynamiques migratoires et diasporiques qui entraînent un changement progressif de l'expérience individuelle et collective du national.

\section{BREF HISTORIQUE DES MIGRATIONS ROUMAINES}

Au cours du XXème siècle et à des époques différentes, plusieurs vagues migratoires ont touché la Roumanie. Les émigrations du début de siècle ont visé les pays du Nouveau Monde. Les habitants de villages entiers de Transylvanie se sont déplacés et installés au Canada, aux États-Unis ou en Argentine aux alentours des années vingt. Entre les deux guerres mondiales, les élites francophones de la Roumanie ont entretenu le mirage de Paris comme centre culturel des Roumains. À cette époque, la formation et la circulation au sein de l'Europe constituaient la norme. La deuxième guerre mondiale mit fin à ce mythe. Dans les deux premières décennies de communisme c'est la fuite, la persécution à outrance et l'exil qui déciment les intellectuels roumains. "La chasse aux sorcières" faisait que les départs de cette période soient à sens unique.

14 Bruneau (Michel), art.cit.

15 Fibbi (Rosita), Meyer (Jean-Baptiste), "Le lien plus que l'essence", Autrepart, 2 (22), 2002 ; Prévélakis (Georges), "Les diasporas comme négation de l'idéologie géographique", communication présentée au colloque international 2000 ans de diasporas, Poitiers, 14-16 février 2002.

16 Sheffer (Gabriel), art.cit.

17 Prévélakis (Georges), "Introduction” (art.cit.).

18 Cf. Cohen (Robin), op.cit. 
Pour apprécier l'ampleur des migrations roumaines après 1989, il est utile de préciser que les dimensions des flux migratoires ne varient pas beaucoup par rapport aux vagues migratoires de l'époque communiste. 288000 personnes ont émigré au cours des années 1980, selon les données du Ministère de l'Intérieur, surtout suite aux accords conclus avec Israël, l'Allemagne, la Hongrie et les États-Unis. La France, l'Australie et le Canada se sont retrouvés aussi parmi les principaux pays de destination. La perte des cadres qualifiés avait déjà pris des proportions non-négligeables ${ }^{19}$ incitant, en 1983, le régime roumain à exiger de la part des candidats à l'émigration le remboursement en devises des coûts de leurs diplômes ${ }^{20}$.

La forte poussée migratoire d'après 1989 , année de la chute du système dictatorial de Ceausescu, s'est exprimée néanmoins par une dynamique extrêmement complexe et variée. Ce qui est nouveau dans ces migrations est la diversité des flux et des opportunités de mobilité ainsi que la liberté de circulation qui fait le retour possible à tout moment. En Europe, l'installation dans la mobilité est le trait de migrations économiques, plutôt circulatoires, des Roumains. Les va-et-vient à l'étranger, parfois dans des situations précaires, plus ou moins clandestines, représentent pour de larges fractions de la population une ressource et une stratégie de survie, réponse au marasme économique dans lequel le pays se débat depuis une décennie ${ }^{21}$.

Mais on assiste en outre à une nouvelle migration, de longue durée, voire même permanente, propre à une population de plus en plus jeune, très qualifiée, consciente d'être en possession d'un capital de formation et de compétences facilement négociables sur un marché global toujours à la recherche de nouveaux talents et spécialistes. Ces flux revêtent deux, voire trois formes particulières : les migrations pour études, la circulation au sein d'un marché de compétences de plus en plus attirant et les migrations permanentes de professionnels, surtout vers les pays traditionnels d'immigration. Il ne s'agit pas de flux nettement différenciés car les passages d'une catégorie à l'autre sont souples (des informaticiens devenus citoyens canadiens n'hésitent pas à retourner sur le marché européen si l'opportunité se présente, de même que des étudiants de $3^{\text {ème }}$ cycle font des projets migratoires de longue durée à la rencontre de l'offre des employeurs occidentaux).

$1920 \%$ des immigrés d'origine roumaine acceptés aux États-Unis de 1980 à 1990 .avaient une formation universitaire (selon Gheorghiu (Mihai Dinu), "La mobilité universitaire internationale, la formation et la reconversions des élites des pays ex-socialistes", in Broady (Donald), Chmatko (Natalia), Saint Martin (Monique de), éds., Formation des élites et culture transnationale, Paris / Uppsala : CSEC / EHESS / SEC / ILU, 1996).

20 Cette exigence a été finalement abandonnée suite aux pressions politiques américaines.

21 Diminescu (Dana), "L'installation dans la mobilité : les savoir-faire migratoires des Roumains", Migration société, 13 (74), 2001 ; Potot (Swanie), "Mobilités en Europe. Étude de deux réseaux migratoires roumains", Sociologie Romaneasca, (2), 2000. 
Les statistiques de l'OIM et de l'OCDE 22, qui se fondent sur des données de l'Institut National de Statistique de la Roumanie (INSSE), estiment à 325000 le nombre des citoyens roumains qui ont émigré de façon permanente de 1990 à 2000. En réalité il serait question de plus de 800 ooo, soit 3,5\% de la population totale de la Roumanie, dont les trois quarts auraient demandé le droit de résidence après leur arrivée dans le pays d'accueil ${ }^{23}$.

Les statistiques officielles des populations d'origine roumaine vivant en dehors de la Roumanie comptabilisent aujourd'hui environ 5 millions de personnes. D'autres estimations avancent la chiffre de 10 millions d'ethniques roumains et d'originaires de Roumanie résidant en dehors des frontières du pays $^{24}$. Ce chiffre englobe les minorités ethniques de Roumains qui, pour des raisons historiques, vivent dans les pays voisins (dont 3,4 millions résident en Moldavie, 500 ooo en Ukraine, 250 ooo dans les pays d'ex URSS, 800000 en exYougoslavie, 150 ooo en Bulgarie, 300 ooo en Macédoine et autant en Albanie, 27000 en Hongrie). Dans les pays occidentaux on estime à environ 1,1 millions de Roumains aux États Unis, 100000 au Canada, 750 ooo en Allemagne (dont 350000 saxons de Roumanie, 130 ooo ethniques roumains, environ 70000 illégaux), 60000 en France ${ }^{25}, 60000$ en Italie ${ }^{26}, 500000$ juifs originaires de Roumanie et environ 80 ooo travailleurs dans la construction en Israël, 30 ००० en Autriche, etc.

Néanmoins, malgré cette présence massive des Roumains en dehors de leur pays, peu d'études ont porté sur leur organisation diasporique. Relativement nouvelle et scindée par la succession des vagues migratoires de motivations diverses, la migration roumaine n'a pas été considérée jusqu'à récemment comme une diaspora. Avant 1989, le contexte historique et géopolitique précédant l'effondrement des systèmes communistes était tel qu'il ne laissait pas une grande marge de manœuvre aux immigrés/exilés. La revendication d'une identité en diaspora restait pourtant leur seule réponse à la politique isolationniste pratiquée par le régime communiste qui niait la reconnaissance de ses ressortissants. La rupture brutale avec la réalité idéologique qu'ils fuyaient aurait pu être de nature à fonder la mémoire d'une diaspora en quête d'identité. Est-ce que ce fût le cas ? La réponse n'est pas simple, les recherches à ce sujet faisant souvent défaut.

22 Les rapports SOPEMI de l'OCDE, éditions 1997, 1999, 2000.

23 Selon la déclaration du responsable de la Direction "Passeports" du Ministère roumain de l'Intérieur, cité par la revue Capital, (13), 29 mars 2001.

24 Selon l'estimation du Ministère roumain des Affaires Étrangères, le département des relations avec les Roumains de l'étranger. http://domino.kappa.ro/mae/politica.nsf/Teme/

25 Appartenant à ce qu'on appelle la vague de la migration historique, la plupart de ces migrants sont devenus des citoyens français.

26 Chiffre auquel s'ajouteraient environ 100000 irréguliers. 
Et si l'exil roumain semble n'avoir pas eu la capacité de se transformer en diaspora, comme certains auteurs le suggèrent, que se passe-t-il pour les nouvelles migrations des Roumains?

\section{RÉSEAUX INSTRUMENTAUX OU CRISTALLISATION D'UNE IDENTITÉ EN DIASPORA?}

Pour décliner la question de la diaspora roumaine, le changement durable voire définitif du lieu de résidence est une condition nécessaire. La seule dispersion n'est pourtant pas suffisante pour constituer une ressource. Elle "devient "positive" dès lors qu'elle est valorisée et revendiquée par les sujets"27. Les stratégies par lesquelles les migrants font valoir leur mobilité à l'âge du numérique sont complexes. L'étude de cas suivant nous permettra de nous interroger sur les effets d'Internet sur la mobilisation des élites roumaines migrantes en diaspora.

\section{Le savoir-circuler collectif ou la constitution d'une mémoire- ressource de la migration de date récente au Canada}

Le Canada, grâce à sa politique d'immigration ciblée, est devenue après 1989 une des destinations principales de jeunes professionnels roumains en quête d'un environnement social et professionnel épanouissant ${ }^{28}$. Entre deux et cinq mille Roumains par année - soit entre $1 \%$ et $2,23 \%$ du contingent annuel d'immigrés réservé aux professionnels ${ }^{29}$ - se sont établis au Canada comme des résidants permanents, avec la perspective attirante d'acquérir la citoyenneté canadienne au bout d'une résidence permanente de trois ans. Probablement, tout autant ont fait les démarches non pas depuis la Roumanie mais depuis d'autres pays occidentaux ou encore résidant déjà au Canada pour

\section{Ma Mung (Emmanuel), art.cit.}

28 Au début des années 1990, les réseaux de compatriotes à l'étranger étaient rares et les opportunités de mobilité favorisaient davantage la formation que le travail. Les migrants arrivaient au Canada suite à l'impossibilité de trouver d'autres niches leur permettant un emploi adéquat dans un pays occidental. Aujourd'hui encore, le Canada est moins fréquemment retenu comme destination de premier choix, pourtant beaucoup de trajets migratoires y aboutissent, grâce à une procédure relativement facile pour obtenir la citoyenneté. La hiérarchisation des espaces d'accueil et de résidence se fait surtout en fonction des opportunités qu'offre le marché des compétences, mais aussi des facteurs subjectifs, de l'accommodation préalable à l'espace migratoire, du soutien des réseaux. L'attraction des pays d'Europe occidentale est ouvertement déclarée dans les discours des individus mais son intangibilité relative est aussi évoquée. Ce n'est que vers la fin des années 1990 qu'un revirement du marché européen ouvre celui-ci aux spécialistes venant de l'Est, Roumains inclus.

29 Selon l'Annuaire statistique de la Roumanie, l'INSSE, éditions 1995-2001 et le rapport Facts and Figures 2001. Immigration Overview du Ministère Citoyenneté et Immigration Canada (CIC) : http://www.cic.gc.ca/english/pdf/pub/facts2001.pdf 
des séjours de durée limitée, pour études ou sous contrat de travail. Ceci fait qu'aujourd'hui, à une migration relativement atomisée qui datait depuis la période communiste, s'ajoute une présence massive de Roumains qualifiés, avec un niveau de formation et un capital d'expertise et d'expérience élevés, fortement concentrés dans quelques centres urbains (Toronto, Montréal, Vancouver $)^{30}$. La fracture qui scinde les différentes générations de migrants selon le contexte politique de leur départ a été à maintes reprises évoquée lors de nos propres enquêtes et elle semble rester un obstacle à la cohésion de la communauté ethnique des Roumains au Canada ${ }^{31}$. Mais la rupture avec le territoire et le pays d'origine s'estompe grâce à la conjoncture géopolitique actuelle et surtout grâce aux moyens de communication et d'information à distance. Les circulations de personnes, d'idées, de biens matériels et symboliques s'intensifient, une "culture du lien" ${ }^{32}$ nourrit les échanges et le déploiement des réseaux dans des espaces transnationaux. La question est comment des migrants installés durablement à l'étranger gèrent leur "coprésence" dans des espaces multiples ? Quels sont les traits spécifiques et les dynamiques communautaires de cette immigration jeune qui nous autoriserait à argumenter en faveur de l'émergence d'une nouvelle diaspora?

En nous intéressant aux stratégies de reproduction des capitaux en situation migratoire, nous avons vite observé une particularité prégnante de la population des migrants roumains après 1989 au Canada. Elle est en possession d'un capital social et culturel élevé dès le départ. Dotés de compétences techniques particulières - car la plupart des migrants sont des professionnels dans le domaine de la technologie de l'information - ces migrants développent et entretiennent des liens sociaux médiatisés par l'ordinateur, dans des espaces sociaux multiples et hybrides. Les pratiques en ligne font partie de leur sociabilité quotidienne, qui se déploie au-delà des frontières étatiques. Outil clé

30 Selon le rapport 2001 de CIC, la Roumanie se trouve depuis 1999 en huitième position dans le "Top Ten" des pays-source pour l'immigration au Canada. Les données partielles du recensement canadien de 2002 révèlent la présence de 60520 Roumains au Canada, dont 21340 à Toronto, 13854 à Montréal, 5750 en Colombie Britannique. Ces chiffres sont à manipuler avec prudence, car il s'agit en effet du nombre des citoyens canadiens ayant déclaré le roumain comme langue maternelle. Elles excluent au moins les migrants temporaires pour travail ou études.

31 Dans la constitution d'une diaspora, les connecteurs entre les vagues migratoires jouent un rôle essentiel. La fracture entre les exilés de la période communiste et les jeunes professionnels parait insurmontable au début des années 1990. Les anciens déconsidèrent ceux arrivés au Canada après 1989 sans assumer des risques, faisant partie d'une élite qui "manque de culture". À l'inverse, le jeune immigré professionnel, motivé par les valeurs du marché, n'apprécient pas à sa juste valeur "l'exilé frustré" qui "a raté sa vie par nostalgie" pour une matrice d’origine idéalisée. Sur un plan plus général, cette situation reflète en effet le renversement des valeurs dans une économie-monde libérée, en partie, des contraintes géopolitiques. À une échelle microsociologique, les liens familiaux et amicaux conservés malgré la rigueur du régime communiste sont brusquement réactivés après 1989 et ont servi de pont d'ancrage pour les nouveaux arrivés.

32 Diminescu (Dana), "Les migrations à l'ère des nouvelles technologies" (art.cit). 
dans le triangle mobilité - communication - connectivité, Internet s'est avéré la meilleure plate-forme pour la mise en commun d'expériences migratoires et la constitution d'un "collectif circulatoire" 33 qui fédère les "savoir-circuler" par l'effet d'émergence d'une mémoire collective de la migration de date récente.

Les données empiriques accumulées en plusieurs étapes entre octobre 1999 et avril 2002 - par entretiens compréhensifs avec des informaticiens à différentes étapes du processus migratoire, en Roumanie et à Toronto, par observation participante (ou netnographie ${ }^{34}$ ) d'un forum de discussion et par l'analyse des discours produits en ligne - nous ont conduit à nous interroger sur le rôle que ce nouvel environnement social acquiert, non seulement dans la reproduction des réseaux migratoires et les processus de reconstruction identitaire, mais aussi dans les pratiques transnationales d'organisation diasporique des Roumains.

\section{TheBans.com ou le catalyseur électronique d'une identité collective}

L'analyse du portail www.thebans.com 35 - une plate-forme web développée par l'instrumentalisation et la mise en scène du capital culturel et de l'expérience migratoire d'un jeune informaticien immigré à Toronto en 1994 - a montré comment la propagation d'une culture de la mobilité s'est formalisée par la concentration de ressources migratoires dans le virtuel. Internet est devenu le support de reproduction d'un modèle archétypal d'expatriation par la mise anticipée en situation migratoire, ce qui facilite une pré-accommodation à distance à la future société d'accueil. Simultanément au déploiement des estratégies migratoires, les dynamiques sociales au cœur du groupe de Roumains au Canada sont-elles aussi multipliées via Internet.

Entreprise aujourd'hui prospère, fondée sur la mise en réseaux des mi-

33 Tarrius (Alain), "Nouvelles formes migratoires, nouveaux cosmopolitismes", in Bassand (Michel), Kaufmann (Vincent), Joye (Dominique), éds., Enjeux de la sociologie urbaine, Lausanne : PPUR, 2001.

34 Pour le chercheur en sciences sociales, Internet n'est plus seulement un outil de recherche, il est également un terrain. Un terrain très dynamique, qui change à coup de click, et qui exige d'être abordé avec les moyens qui lui sont propres. Il y a deux dimensions à saisir, soit un côté "information" et un côté "communication" de l'Internet. Car si Internet fait circuler des manières de dire qui sont des véhicules de formes de pensée, chargées culturellement et idéologiquement, la production des discours s'accompagne d'une utilisation complexe. Et pour restituer les usages il ne suffit pas d'observer les pratiques en ligne, il s'agit d'aller plus loin et de se mettre à proximité des (migrants) internautes pour voir comment ils choisissent et interrogent les sites web, avec quelle utilisation de l'information disponible en ligne. De cette manière on saisira davantage l'hybridation de formes des sociabilités et l'impact des e-pratiques sur le quotidien. Le piège d'une pensée simplificatrice marquée par le déterminisme technique pourrait être ainsi évité. La "netnographie" des groupes virtuels, c'est-à-dire l'observation participante aux chats et aux forums des discussions, tout en constituant une nouvelle forme de "champ" anthropologique, repose pourtant sur peu d'études empiriques et demande, pour cela, une forte distance critique par rapport à son objet.

35 Nedelcu (Mihaela), "L'instrumentalisation de l'espace virtuel par une communauté des professionnels immigrés : vers une nouvelle forme d'organisation diasporique ?", Autrepart, 2 (22), 2002. 
grants roumains, TheBans visibilise la communauté roumaine à Toronto. En valorisant les compétences professionnelles, techniques et sociales de son initiateur, ce projet - qui est à la fois carrefour des réseaux migratoires et professionnels, espace de sociabilité des migrants mais aussi incubateur et vitrine communautaire - a propulsé celui-ci en position d'interface (d'abord informelle et non institutionnalisée) de la communauté ethnique roumaine.

\section{QUeL MYTHE D'ORIGINE POUR QUELLE IDENTITÉ ?}

Le rapport à la Roumanie, tel qu'il transparait dans les échanges en ligne, est complexe. Il n'est pas seulement d'ordre familial et sentimental, mais également économique et politique. L'expression d'une conscience de soi, déclinée par rapport à l'appartenance nationale, culturelle et linguistique d'origine, est présente dans la structure même de ce $\operatorname{site}^{36}$. La volonté d'affichage d'une appartenance ethnique assumée et de l'affiliation à la culture roumaine se traduit par la mise en scène d'une image valorisante de la Roumanie à travers le site 37 .

Internet s'avère un environnement dual car tout en étant permissif à la démultiplication du collectif (tribune de l'individu), il permet la production d'une centralité (collective). TheBans fabrique une mémoire collective de la migration de date récente (par la transmission des savoirs migratoires) mais aussi une mémoire de l'origine partagée. Par une permanente actualisation des marqueurs symboliques d'appartenance (langue, religion, traditions, fêtes, vie culturelle), TheBans maintient vif le sentiment identitaire. On parle en général d'une diaspora selon la persistance de la communauté au-delà de la dispersion dans l'espace. Dans le virtuel, on fabrique une communauté transnationale d'intérêts - conjoncturels ou durables - là où l'unité faisait défaut à l'origine. Des personnes qui ont en commun l'expérience de l'errance trouvent intérêt à renforcer l'imaginaire d'une continuité (culturelle, sociale et même psychologique). Les forums de discussions, espaces de négociation et d'expression d'identités individuelles multiples, juxtaposables et difficilement recomposables

36 Structuré autour des deux pôles de la migration (roumain et canadien), le réseau de websites véhicule toute information touchant à la vie des Roumains à Toronto : annonces de spectacles, expositions, concerts, journaux, programmes TV et chaines radio en roumain, promotions d'entreprises ethniques de compatriotes, offres d'emplois, etc. Il contient plusieurs rubriques : Romanian world (forums, nouveautés, la Une, coutumes roumaines, informations pratiques) ; Romanian universe (culture, religion, histoire, langue) ; Toronto (Romanian news, l'école roumaine de Toronto, artistes roumains à Toronto, Romanian business network); Directories (liste de liens vers des sites et médias roumains, albums de photos) ; IT Consulting (présentation de l'équipe et des services offerts par The Bans). Une base de données en ligne (RomUniv) et un forum de discussions à domaines multiples rendent visibles les professionnels migrants.

37 L'impact de cette forte affirmation identitaire ne tarde pas à se manifester. Des agents touristiques, des entrepreneurs canadiens, des associations humanitaires ou des investisseurs potentiels trouvent en TheBans un partenaire de discussion averti. 
en situation migratoire, font des participants aux échanges des producteurs à part entière des modèles identitaires collectifs. Le processus de "fabrication d'une continuité généalogique à travers la création d'une mémoire / histoire propre à la diaspora" ${ }^{38}$, qui accentue son caractère d'artefact, est d'autant plus conforté que l'émergence de nouveaux territoires "virtuels" permet de penser la dispersion des individus et leurs trajectoires comme un processus unitaire dans l'espace.

Le mythe d'origine, quant à lui, n'est plus le reflet d'une mémoire douloureuse de la rupture violente avec le territoire d'origine (guerre, dispersion forcée) car les logiques de mobilité sont multiples. Plus entraînés à la circulation inhérente à un marché globalisé, dynamisés par le succès économique de leur mobilité, les jeunes professionnels assument relativement sans difficulté le projet migratoire. Ils ne sont toutefois pas exemptés des coûts psychologiques de l'intégration. Les débats vifs qui animent les forums de discussions à ce sujet en sont la preuve. L'impuissance face au marasme économique du pays d'origine, à laquelle s'ajoute la mémoire encore fraîche d'un communisme atrophiant qui coupait tout espoir de mobilité, se substitue à l'avènement violent de la rupture que provoquait la dispersion. Néanmoins, ce n'est pas principalement le passé qui unit ces migrants mais plutôt la projection dans un futur à parfaire. L'identité collective se cristallise autour d'une expérience commune du présent et sur des projets d'avenir qui s'articulent sur des horizons spatio-temporels étendus que les migrants élaborent à travers leurs parcours et pratiques. Le rapport traditionnel des diasporas au temps, comme à l'espace, est ainsi modifié.

\section{REgROUPEMENT ASSOCIATIF OU LE CAPITAL SOCIAL COLleCtIVEMENT POSSÉdÉ \\ "Unde-i unul nu-i putere..."39}

Le Canada, en tant que pays d'immigration, incorpore dans son projet de légitimité l'idée de diversité. Il encourage la préservation d'une identité d'origine, individuelle (pratiques alimentaires, religieuses, etc.) et collective (pratiques culturelles, associatives). La société canadienne offre l'environnement socio-politique permissif au particularisme, et donc propice au développement d'une culture de diaspora (école, pratiques religieuses, conservation des traditions, pratiques matrimoniales, fetes, etc.). Former une diaspora peut devenir ainsi un projet identitaire qui sert d'outil d'intégration à la société d'accueil. Sans exacerber la positivité des conséquences de la sociabilité en ligne, on doit

38 Ma Mung (Emmanuel), art.cit.

39 “Oú il n'y a qu'un, il n'y a pas du pouvoir...". Motto extrait du Forum, quí singularise les interventions de M., l'initiateur de TheBans, au forum Romania online en définissant ainsi son identité virtuelle et son credo. Ce motto est symboliquement chargé car il s'agit d'un extrait de l'hymne roumain de l'unité, qui a marqué l'union historique des trois provinces roumaines le 1er décembre 1918. 
remarquer qu'Internet démocratise la participation civique tout en offrant des outils pour la préservation d'un héritage culturel d'origine (langue, traditions, éducation) et le développement des réseaux. Les loyautés développées sont duales, vers les pays d'accueil et d'origine. On assiste à une institutionnalisation progressive du communautaire, un minimum de stabilité des échanges culturels et politiques se formalisant au cœur de la société d'accueil. En disposant de ce que Cohen appelle "an advantageous occupational profile" $4{ }^{\circ}$, les membres de cette diaspora à trait technocratique profitent d'une niche créative d'organisation dans un pays d'accueil ouvert et tolérant.

Les actions de mobilisation sur la "Toile", entamées à partir des forums de discussions du portail virtuel TheBans, ont débouché sur des processus associatifs plus ou moins institutionnalisés. Le capital social dont disposent les participants aux échanges est réinvesti et, par la mise en commun de ressources, deviendra un "bien collectif". Des relations nouées dans l'espace virtuel se sont naturellement prolongées dans le quotidien, des groupes qui s'organisent en "comités d'accueil" de nouveaux arrivés se réunissent régulièrement, des initiatives entrepreneuriales et touristiques se concrétisent. L'imbrication réelvirtuel témoigne d'un continuum social qui ne fait que différer d'elle-même une réalité sociale complexe. La création d'une école roumaine à Toronto en automne 2001 a couronné ce processus de cristallisation communautaire via Internet. Accueillant cent soixante dix enfants, cette école de samedi est conçue comme le noyau d'un nouvel incubateur communautaire qui gravite autour de The Bans. Les cours des enfants représentent pour les parents un prétexte pour se rencontrer, s'informer, monter des projets économiques et communautaires ou simplement s'amuser ensemble. Le projet d'un club et d'un centre d'affaires roumains à Toronto est né en prolongement de ces rencontres hebdomadaires. La cohésion de la communauté est ainsi renforcée par le regroupement associatif.

La dimension professionnelle, structurante des processus migratoires et communautaires des Roumains au Canada, oriente également les intérêts de cette nouvelle diaspora, d'essence technocratique. En possession d'un capital professionnel, social et culturel élevé, les migrants semblent avoir trouvé dans les nouvelles technologies un outil d'innovation sociale qui leur permet de raccourcir les procédures d'intégration tout en développant des réseaux professionnels, culturels et sociaux qui franchissent les limites géopolitiques des nations ainsi que les logiques étatiques. Sans avoir hérité des réseaux marchands ou intellectuels fortement structurés d'une époque antérieure (comme c'est le cas des Italiens, des Grecs, des Chinois), The Bans construit également un espace économique - sous-tendu par les réseaux professionnels déployés dans 
le virtuel. The Bans Romanian Business Network in Canada est un réseau qui fait la promotion des affaires ethniques des Roumains - Canadiens. L'efficacité de ce réseau (d'informaticiens, médecins, restaurateurs, graphistes, transporteurs, agents touristiques, musiciens, conseillers financiers, etc.) est renforcé par la propagation du principe "de bouche à oreille" à large échelle.

Le projet d'une association qui institutionnaliserait les relations sociales, professionnelles et économiques au sein de la communauté roumaine à Toronto s'est concrétisé en décembre 2002 par la création de l'Alliance des Roumains Canadiens (ARC). La particularité de l'ARC, par rapport à d'autres organisations fondées sur des bases ethniques, est qu'elle représente l'officialisation et la matérialisation d'une communauté de facto cristallisée au sein de "l'agora électronique" et qu'elle concrétise les compétences de management communautaire développées et reproduites dans les échanges médiatisés par ordinateur. Ayant comme buts déclarés de "préserver et propager les valeurs culturelles roumaines, de faciliter l'intégration personnelle, professionnelle et sociale des nouveaux-arrivés dans la société canadienne et de stimuler le dialogue intra-communautaire" 41 , l'ARC reflète l'orientation activiste de cette nouvelle communauté de migrants. D'ailleurs, la structure-même de l'association témoigne d'une mobilisation et d'une mise en scène efficaces des ressources professionnelles et sociales que ses membres incorporent ${ }^{42}$. La devise de l'ARC - "Ensemble on réussit !" 43 - indique une fois de plus le caractère instrumental du regroupement communautaire.

L'esprit d'entreprise et les circulations des hommes, des marchandises, des idées, des savoir-faire et des savoir-circuler 44 nourrissent en conséquence l'espace transnational de cette diaspora technocratique en germe. Au moins deux questions subsistent : ce ressort efficace pour activer la production d'une élite au service d'une intégration et d'une capitalisation au sein de la société d'accueil, sera-t-il déclencheur de la mise en réseau des élites émigrées en vue d'un réinvestissement en Roumanie ? En plus, l'homogénéité professionnelle contribue certainement à consolider l'identité collective, mais on pourrait se demander dans quelle mesure ne risque-t-elle pas de rester exclusive. 45

41 http://www.arcweb.ca

42 Il est à noter l'organisation par départements et services de l'ARC (développement professionnel/business, intégration, services socioculturels, finances/membres et marketing/relations publiques), avec un organigramme détaillé, qui rappelle le background et les compétences d'entrepreneurs de ses initiateurs.

43 "Impreuna reusim !", en roumain.

44 Tarrius (Alain), "Territoires circulatoires et espaces urbains", Annales de la Recherche Urbaine, (59-60), 1993.

45 Les professionnels font corps autour de l'école et de l'ARC, tandis que les personnes moins qualifiées se retrouvent davantage autour de l'église orthodoxe. 


\section{Projets Politioues des migrants et des États-Nations}

La transmission intergénérationnelle des repères fondateurs de cette mémoire de l'origine commune, réelle ou imaginaire, reste un processus qui exige d'être inscrit dans le temps. La cristallisation même d'une conscience d'existence en diaspora suppose une longue durée. L'équilibre des processus que nous venons de décrire est fragile car la dimension temporelle fait défaut. Pourtant un projet de réinvestissement de la $2^{\text {ème }}$ génération apparaît déjà dans l'intention des membres de cette communauté et des participants aux forums de discussions. Par leurs propres actions tournées vers l'avenir ils n'hésitent pas à projeter une deuxième génération fortement ancrée dans les deux univers sociaux, roumain et canadien. Libérée des frustrations des parents, "canadienne" par naissance mais héritière d'un bagage culturel d'origine maintenu vif par l'école roumaine, elle serait connectée à la réalité roumaine dans un espace social de co-présence dans lequel les nouvelles technologies jouent un rôle-clé.

Non moins intéressante est la position d'une génération qui vit indirectement la migration, la génération des parents de ces jeunes professionnels migrants. Ils contribuent à perpétuer la "présence en absence" de leurs enfants en Roumanie, et non pas par la diffusion nostalgique des nouvelles les concernant mais d'une manière parfois beaucoup plus pragmatique : en s'impliquant à diriger leurs affaires sur place. Leur existence est ainsi radicalement bouleversée, non pas par la rupture mais par la continuité des rapports déplacés dans un espace transnational qu'ils ne sont pas forcement entraînés à maîtriser46.

Facteur de développement local, les initiatives entrepreneuriales des migrants permettent souvent à la famille restée au pays de s'assurer une existence décente. C'est par exemple le cas des antennes roumaines des petites entreprises informatiques qui assurent la sous-traitance des contrats en Roumanie, mais aussi des agences touristiques, immobiliers ou de transport, gérés par des parents des migrants. Ce genre d'entreprises, souvent familiales, prend en charge une gamme large de services spécialisés destinés à une population qui déploie ses compétences dans un espace étendu.

La dimension historique d'une diaspora dans son sens paradigmatique est ainsi contournée. Les diasporas modernes s'avèrent plutôt des processus dyna-

46 Le projet d'un club roumain à Toronto vient aussi à la rencontre des besoins des parents des migrants. Présents au Canada quelques mois par année, ils doivent s'approprier un univers qui leur est étranger et qui exige des compétences - linguistiques, de mobilité, etc. - qu'ils ne possèdent généralement pas. Dans le cadre du département socioculturel de l'ARC, un club des grands-parents a été récemment inauguré. Des cours d'anglais et d'utilisation de l'ordinateur et de l'Internet ont été envisagés pour éviter le dépaysement. D'ailleurs l'usage de l'e-mail est devenu l'outil quotidien qui assure la participation à distance à la vie de la famille dispersée de même que lire la presse roumaine en ligne constitue une pratique courante des parents pendant le temps qu'ils résident au Canada. Ces moyens n'entretiennent pas l'illusion "d'être ensemble" mais représentent une autre façon de l'être. 
miques, fluides et polycentriques, ayant leurs racines dans une localité donnée conjuguée au présent. Un projet politique activiste leur est inhérent. Si un investissement politique des migrants dans les deux sociétés est moins explicitement manifesté dans les échanges sur les forums, pourtant une préoccupation pour l'orientation politique de la Roumanie est quasi-présente. D'ailleurs, dans les témoignages des migrants, la raison politique est souvent invoquée, explicitement ou non 47 . La participation des membres de l'équipe TheBans en tant qu'observateurs auprès du consulat roumain de Toronto lors des élections législatives de 2000 et la mobilisation pour une participation engagée, encouragée sur le website, témoignent d'une préoccupation non-dissimulée pour l'avenir de la Roumanie. D'autres projets politiques prennent forme autour de l'initiateur de TheBans en Roumanie et au Canada. Certains des professionnels qui l'entourent envisagent de le propulser dans l'administration torontaise ${ }^{48}$. La reconnaissance de son rôle-clé au sein de la communauté des professionnels au Canada a également été affirmée à travers son invitation au forum "Les Roumains dans la science contemporaine" organisé en 2000 en Roumanie par les représentants de la société civile et du gouvernement roumain.

Pour un pays comme la Roumanie, la volonté du pays d'origine d'intégrer ses communautés expatriées et de s'approprier le capital d'influence de ses ressortissants au sein de la société d'accueil est relativement nouvelle. Des changements législatifs significatifs 49 font preuve de la volonté de l'État-nation "à considérer sa diaspora comme l'extension à l'étranger de sa propre nation" 50 . Néanmoins ce programme politique reste souvent intentionnel de

47 La dernière "minériade" en 1998 a déterminé beaucoup de jeunes qualifiés à émigrer définitivement. De même que le désenchantement consécutif aux prestations politiques décevantes des partis "historiques" qui ont entraîné le retour au pouvoir du président Iliescu en 2000.

48 D'ailleurs il a été invité dans le parlement de la province d'Ontario, avec d'autres représentants de la communauté roumaine, à l'occasion de la nomination de la Roumanie comme futur membre de l'OTAN. Cette opportunité a été prise pour donner davantage de visibilité aux roumains-canadiens et à leur rapport aux deux pays.

49 Après la chute du communisme, la politique roumaine fut réorientée (au moins dans l'intention) vers un traitement non - différencié des citoyens roumains du pays et de l'étranger (au niveau du droit de vote, de propriété, etc.), avec un intérêt affirmé d'associer un certain nombre d'experts roumains expatriés au revirement de l'économie nationale. Ces processus n'ont cependant pas abouti sur une politique cohérente et durable d'activation de la diaspora. En 2000, un "appel vers tous les Roumains du pays et du monde" a été conjointement lancé par le patriarche de l'église orthodoxe roumaine, le président de la république et l'ancien roi de la Roumanie. Une mobilisation de toutes les forces politiques, financiers et économiques était ainsi visée par cette triple autorité.

5o Bruneau (Michel), art.cit. 
même que les intérêts des migrants et des diasporas ne coïncident pas forcements avec ceux de leur pays d'origine ${ }^{51}$.

Il est certain que la question des retours se décline différemment qu'il y a une vingtaine d'années. Sans être impératif car possible à tout moment, le retour n'est plus ni illusoire ni nostalgique. Il fait plutôt partie du projet de mobilité, en se concrétisant dans les va-et-vient multiples entre pays d'accueil et d'origine et surtout dans ses formes non-physiques et non nostalgiques: transfert de connaissances et de fonds, relations économiques, etc.

On peut se demander si, par leur contribution au développement culturel et économique, par le transfert d'expertise et par une reproduction à distance des idées démocratiques et de la multiculturalité, les diasporas ne seraient pas le paradigme de l'avenir de la politique à l'âge de la mondialisation . La multicitoyenneté 52 permet déjà l'exercice du droit électif par rapport à au moins deux États et le fonctionnement en diaspora - qui s'avère ainsi un processus non pas seulement transnational mais aussi trans-étatique 53 - remet en cause le rapport à l'Etat national et la simultanéité culturelle, sociale et politique de ses membres.

\section{Multiplication des pratiques diasporiques médiatisées par ordinateur}

TheBans promeut des réseaux transnationaux de Roumains hautement qualifiés. C'est le cas de RomUniv, une base de données propre qui regroupe sept à huit mille Roumains hautement qualifiés. À cette "académie virtuelle de l'intelligence roumaine dans le monde" ont adhéré principalement des professionnels et des chercheurs roumains émigrés dans quarante pays. Internet s'a-

51 Par exemple, un projet appelé "Return to Romania - Career Development Project" et géré par l'International Research and Exchange Board (IREX), vise le retour au pays des jeunes professionnels roumains qui se trouvent actuellement dans les universités américaines ( 1807 étudiants roumains étaient inscrits pour l'année universitaire 1999 / 2000, dont 1200 en programmes de troisième cycle). Lors de l'entretien que nous avons eu avec la directrice du projet de l'IREX au printemps 2000 , elle estimait que seulement $10 \%$ des Roumains qui étudient à l'étranger rentrent en Roumanie. Les raisons invoquées par les étudiants sont: les difficultés à trouver un emploi adéquat sur le marché roumain, les salaires très bas et le risque de rater l'investissement dans leur formation. Le but de ce programme est précisément d'identifier des emplois appropriés à leur niveau de formation. Mais les quelques réponses reçues de la part des étudiants sont qualifiées d'irréalistes par la directrice, car les jeunes demandent des salaires de 60 75000 dollars / an. En contact avec la réalité et le marché mondial des compétences, les jeunes professionnels sont devenus conscients de leur valeur et de la possibilité de fructifier l'investissement psychologique, temporel et financier dans leurs études. Ils connaissent les alternatives qu'ils ont et le pays ne peut pas compter sur leurs compétences sans leur garantir au moins la reconnaissance du statut et les moyens décents de vivre.

52 Une grande partie des migrants de la nouvelle vague ont gardé leur nationalité roumaine.

53 Faist (Thomas), "Dual citizenship as Overlapping Membership", International Migration and Ethnic Relations, (3), 2001 (www.bit.mah.se/imer/publications) ; Sheffer (Gabriel), "A political Approach to the Ethno-National Diasporism", communication présentée au colloque international 2000 ans de diasporas, Poitiers, 14-16 février 2002. 
vère l'outil approprié pour la connexion dans un espace transnational, en permettant de retrouver des anciens amis ou collègues, d'échanger des idées, de former des groupes de discussions, de se mettre en réseau.

Les fonctions remplies par l'espace virtuel dans le cas analysé sont multiples : d'abord expressives et instrumentales, mais aussi associatives et intégratives, car le virtuel a une valeur performative. TheBans, exemplaire par son rôle d'incitateur de pratiques locales et transnationales et par son impact communautaire au quotidien, n'est pourtant pas un exemple isolé. D'autres forums ou listes de discussions, parfois orientés exclusivement sur le processus de migration, modérés et médiatisés, remplissent des fonctions similaires. Escape from Romania est un groupe de discussion initié par un ancien participant au forum Romania online. Au 4 mars 2003, ce groupe avait non moins de 2172 membres et 32615 messages, ciblés autour du thème de la migration, ont été envoyé depuis la date de sa création (le 13 août 2000). Actuellement, un noyau des membres de ce groupe s'est constitué en comité d'accueil pour les nouveaux arrivés. Ils se retrouvent régulièrement à Toronto et des communications en duplex sont organisées à distance, entre Bucarest et Toronto, à l'aide de webcams. BC Romanian Community Center, une nouvelle association de Roumains à Vancouver, se rend elle aussi visible à travers son site Internet. Et les exemples se multiplient, avec des variantes bien sûr, au Canada, aux États-Unis 54 et, timidement, en Europe. Le potentiel de ces pratiques semble être cumulatif, Internet étant le milieu adéquat à la mise en réseau des noyaux diasporiques.

\section{Per aspera AD ASTRA}

Un autre exemple, dont l'analyse est pour l'instant moins approfondie, suggère une fois de plus le potentiel d'Internet en tant que plate-forme programmatique de réinvestissement des élites expatriées dans le pays d'origine. Il s'agit d'Ad Astra : An Online Project for the Romanian Scientific Community, un projet de mise en réseau des scientifiques roumains, du pays et de l'étranger, né comme initiative informelle en prolongement du premier forum des jeunes roumains formés à l'étranger.

Ad-Astra est devenu le lieu de rencontre des jeunes scientifiques roumains engagés dans la production scientifique mondiale car actifs dans des laboratoires de recherche aux États-Unis, en Suède, France, Allemagne, Australie, Grande Bretagne, ... Ce projet se concrétise par une plate-forme d'échanges scientifiques et de mise en commun d'expertise et de compétences. Un journal scientifique en ligne, une base de données Who's who dans la science rou-

54 www.romanianamericans.org visibilise The Congress of Romanian Americans, une organisation qui depuis 1991 institutionnalise la reconnaissance de l'exil roumain aux États-Unis en tant que facteur de lobby auprès de l'administration américaine. 
maine 55 , par domaine scientifique, un calendrier des événements scientifiques à jour, font la carte de visite d'une communauté scientifique déterritorialisée. Ces jeunes doivent leur succès et leur rôle de catalyseur de la communauté scientifique roumaine à la sphère transnationale. Des 15 éditeurs en ligne, 2 résident en Roumanie, 6 aux États-Unis, 2 en France, 1 en Suède, 1 en Suisse, 1 en Espagne, 1 en Australie, 1 en Grande Bretagne. Mais si "la science est la patrie des scientifiques" 56 , cette "agora scientifique" se mobilise et s'exprime par rapport à une centralité culturelle. L'orientation militante de cette communauté se nourrit dans l'attachement exprimé par l'aspiration à exercer une influence dans le développement du pays d'origine. Si tout le travail d'édition se déroule par courrier électronique - la plupart des éditeurs, contributeurs ou membres de la communauté en ligne n'ayant pas de rapports sociaux de faceà-face - cela n'a pas empêché que ce réseau ait obtenu une personnalité juridique en Roumanie, en devenant ainsi partenaire légitime des acteurs institutionnels agissant dans le monde de la recherche mais aussi dans la sphère de la société civile en Roumanie.

L'orientation activiste de ce réseau scientifique en ligne l'inscrit dans une option e-diaspora à effet performant. Le retour physique du cerveau n'est plus indispensable, ce qui est essentiel c'est qu'il soit connecté, qu'il participe et souscrive activement au travail collectif du réseau. La Roumanie pourrait ainsi avoir accès non seulement aux connaissances incorporées mais aussi aux réseaux socioprofessionnels des scientifiques expatriés et aux contenus véhiculés par ceux-ci57. La connectivité pourrait être l'outil-clé pour contourner la perte du potentiel de développement incorporé par ses scientifiques expatriés, à condition d'un effort social, politique et technique à long terme.

55 L'inscription dans cette base de données est acceptée selon les critères de production scientifique de la communauté internationale. Des 143 membres, 48 sont aux États-Unis, 46 en Roumanie, 12 en France, 8 en Allemagne, 5 au Canada et en Grande Bretagne, 3 en Grèce, Pays Bas et Suisse, 2 en Italie, Australie et Moldavie, 1 en Suède, Belge, Irlande et Autriche (selon l'interrogation de cette base le 27 novembre 2002). Sans refléter la dispersion des scientifiques roumains dans le monde, cette répartition met en évidence l'attrait des États-Unis en tant que pôle d'excellence scientifique.

56 Morokvasic (Mirjana), "La mobilité des élites scientifiques de l'Autre Europe : exode ou circulation?", Revue d'études comparatives Est-Ouest, (3), 1996.

57 Meyer (Jean-Baptiste), Charum (Jorge), "La "fuite des cerveaux" est-elle épuisée? Paradigme perdu et nouvelles perspectives", Cahiers des Sciences Humaines, 31 (4), 1995. 


\section{LE RÉFÉRANT NATIONAL DE L'ESPACE TRANSNATIONAL DES DIASPORAS}

Ces nouveaux migrants sont tous des transmigrants dans le sens défini par N. Glick Schiller, L. Basch et C. Szanton Blanc : "transmigrants are immigrants whose daily lives depend on multiples and constant interconnections across international borders and whose public identities are configured in relationship to more than one nation-state" 58 . Ils se caractérisent par le fait de construire et d'entretenir au quotidien des connections entre eux et avec le pays d'origine au niveau familial, professionnel et institutionnel, en s'impliquant activement dans l'échange de biens matériels et symboliques, dans la reproduction de relations sociales et d'expertise dans les deux sociétés, d'origine et d'accueil. Leur existence quotidienne est ainsi reliée avec plus d'une société, les réseaux qui trouvent support dans les TIC étant denses et couvrant des longues distances. "Le rapport au territoire (...) est remplacé par un sentiment d'extraterritorialité"59 sur lequel repose la capitalisation de ressources diasporiques dans l'espace virtuel.

Tant TheBans qu'Ad Astra accélèrent une consolidation institutionnalisable des entités sociales qui ont pris forme à partir des réseaux sociaux soutenus par ordinateur. Les TIC sont à la fois l'incitateur et le régulateur de la "diasporisation", en propageant les processus de cristallisation d'une conscience en diaspora par une combinaison entre cosmopolitisme et collectivisme ethnique $^{60}$. Dans un rapport paradoxal, Internet joue un rôle d'unificateur de ces populations en dispersion, tout en déterritorialisant leurs pratiques.

Et si la mobilité est le trait essentiel de la constitution et de la mobilisation de cette nouvelle diaspora, celle-ci se cristallise toujours par rapport à trois référents : local, national (qui continue à nourrir la conscience d'être une minorité) et transnational. Internet, en facilitant la mise en réseau et une représentation de soi cohérente, produit un espace de mobilité englobant qui est en soi le "non-lieu de la diaspora" 61 ; mais le trans ou plutôt le meta-national acquiert du sens seulement par rapport à un local quotidien et un national de référence. La diaspora se reproduit, dans ce cas, dans une communauté transnationale d'intentions et d'intérêts qui subsiste surtout grâce à la mobilisation locale des ressources et à l'identification nationale.

58 Glick Schiller (N. G.), Basch (L.), Szanton Blanc (C.), "From Immigrant to Transmigrant : theorizing transnational migration", Anthropological Quarterly, 1994.

59 Ma Mung (Emmanuel), "La désignation des diasporas sur Internet", Hommes \& Migrations, (1240), 2002.

60 Cohen (Robin), op.cit.

61 Ma Mung (Emmanuel), "Non-lieu et utopie : la diaspora chinoise et le territoire", Espace géographique, $23(2), 1994$. 


\section{CONCLUSION}

La question centrale de cette étude, telle que nous l'avons présentée au début, était de savoir si la diaspora reste un concept encore approprié dans le contexte des dynamiques politiques, migratoires et économiques radicalement nouvelles à l'âge de la mondialisation et du numérique.

Nous avons vu qu'une migration très récente peut cristalliser rapidement les traits d'une diaspora. Néanmoins, si les TIC sont à l'origine d'une "diasporisation" accélérée des communautés roumaines, la persistance de ce processus reste dépendante d'un certain nombre de facteurs. La nouveauté réside dans l'effet d'activation des diasporas à travers l'espace virtuel, processus davantage décentralisé et autonome. Mais la spécificité de la population concernée - citadine, avec un niveau de compétences professionnelles, culturelles et sociales élevé - rend la généralisation de ce phénomène hasardeuse. Au Canada, les migrants roumains internautes sont une majorité. Ils s'approprient les technologies au quotidien ce qui fait d'Internet le "levier" de l'organisation communautaire au sein du pays d'accueil et de la transformation à l'intérieur du pays d'origine. Il est pourtant difficile d'estimer l'effet de mise en réseau et de “diasporisation" de ce même outil dans les mains des populations avec des caractéristiques socio-professionnelles différentes (non qualifiées ou d'origine paysanne par exemple). L'accès aux technologies est encore un élément d'exclusion ${ }^{62}$ et l'effet de manipulation idéologique à distance reste d'autant plus dangereux qu'une illusion de participation démocratique est facilement entretenue. Malgré ces inconvénients, le brassage des modèles culturels - phénomène à deux pôles - est un processus incontournable, Internet façonnant différemment l'expérience culturelle nationale de nouvelles générations.

Un autre aspect qui contribue à une mise en perspective différente du concept de diaspora est le rapport au facteur temps. Le synchronique et non plus le diachronique devient le temps de conjugaison des nouvelles entités diasporiques. Les diasporas modernes désignent des processus dynamiques ancrés dans le présent et souvent tournés vers l'avenir. En développant une logique propre de propagation, elles valorisent la multi-appartenance de leurs membres. Économiques et culturelles, les actions des diasporas ne sont pas moins politisées, en contribuant à l'intégration des logiques étatiques dans des processus par essence transnationaux. Et finalement, dans ce contexte extrêmement moderne, ce qui nous encourage davantage à plaider pour l'émergence d'une diaspora c'est la présence incontournable du projet national. Malgré les discours prophétiques sur la dissolution des État-Nations dans un 
espace mondialisé de gouvernance et d'échanges, le rôle et le devenir de la nation ne semble pas avoir dramatiquement changé. L'heure du post-national et de la citoyenneté supra-étatique ${ }^{63}$ n'est pas encore arrivée, le transnational se définit toujours par rapport à un national structurant. 